\title{
Deterministic Global Optimization of Molecular Structures Using Interval Analysis
}

\author{
Youdong Lin and Mark A. Stadtherr* \\ Department of Chemical and Biomolecular Engineering \\ University of Notre Dame, Notre Dame, IN 46556, USA
}

November 16, 2004

(revised, April 18, 2005)

*Author to whom all correspondence should be addressed. Phone: (574) 631-9318; Fax: (574) 631-8366;

E-mail: markst@nd.edu 


\begin{abstract}
The search for the global minimum of a molecular potential energy surface is a challenging problem. The molecular structure corresponding to the global minimum is of particular importance since it usually dictates both the physical and chemical properties of the molecule. The existence of an extremely large number of local minima, the number of which may increase exponentially with the size of the molecule, makes this global minimization problem extremely difficult. A new strategy is described here for solving such global minimization problems deterministically. The methodology is based on interval analysis, and provides a mathematical and computational guarantee that the molecular structure with the global minimum potential energy will be found. The technique is demonstrated using two sets of example problems. The first set involves a relatively simple potential model, and problems with up to 40 atoms. The second set involves a more realistic potential energy function, representative of those in current use, and problems with up to eleven atoms.
\end{abstract}

Keywords: Global optimization; Molecular structure; Interval analysis; Potential energy 


\section{Introduction}

The search for the global minimum of a molecular potential energy surface (PES) is one of the most challenging problems in computational chemistry and applied mathematics. For a given molecule, there are typically many possible conformational geometries (structures). The conformation corresponding to the global minimum of the PES is of particular importance since it dictates both the physical and chemical properties of the molecule in the great majority of cases. The existence of a very large number of local minima, the number of which often increases exponentially with the size of the molecule, makes the global minimization problem extremely difficult.

Traditional local optimization methods, such as gradient-based search algorithms, will converge only to local minima. While brute force multistart approaches using local methods may be used, this is often impractical due to the large number of local minima that exist, and, moreover, there is no guarantee that the global minimum will be found. Thus, a large number of alternative methods have been proposed for finding the global minimum of the PES. Most of these methods can be categorized as stochastic methods. Stochastic approaches rely on probabilistic methods to find the global optimum. Since random elements are involved, there are no theoretical guarantees that these methods will always find the global solution. Methods in this category include genetic algorithms, ${ }^{1}$ simulated annealing, ${ }^{2}$ Monte Carlo approaches ${ }^{3}$ etc. Another main category of methods are the smoothing or deformation methods. Here the shape of the PES is altered in an attempt to reduce the number of local minima and make the problem easier. Methods in this category include the diffusion equation method ${ }^{4}$ and distance scaling methods. ${ }^{5}$ Because of the difficulties involved in reversing the deformation to recapture the true global minimum, these methods also provide no guarantee that the global minimum of the original PES will be found. A final main category of 
methods are those that are truly deterministic. Unlike other methods, the deterministic methods ${ }^{6}$ can offer some level of guarantee that the global minimum will be found. In this category are the $\alpha$-BB algorithm ${ }^{7,8}$ and the interval approach recently given by Lavor. ${ }^{9}$ Both of these techniques employ a branch-and-bound strategy that effectively searches the entire conformational space in order to find the global solution. For a survey of these and other approaches, a number of good reviews are available. ${ }^{10-14}$

The key step in each iteration of a branch-and-bound method is to determine a lower bound on the potential energy over some subdomain of the search space. This lower bound is then compared to a known upper bound on the global minimum of the PES, a bound that can be obtained and updated simply by function evaluations or by the use of local minimization techniques. If the lower bound on the potential energy over the current subdomain is greater than the known upper bound on the global minimum, this proves that the global minimum cannot be in the current subdomain and so it need not be further searched. Otherwise, if the lower bound on the current subdomain is less than the known upper bound, then the current subdomain is partitioned into smaller subdomains over which tighter lower bounds can be generated. The efficiency of a branchand-bound method depends on a number of factors, including how quickly a good upper bound can be identified and how tight the lower bounds are. In the $\alpha$-BB algorithm, convex underestimating functions are used to obtain the lower bounds on the potential energy. However, whether or not these are valid bounds depends on the proper choice of a parameter $(\alpha)$. Methods exist, ${ }^{15,16}$ based on an interval representation of the Hessian matrix, that can be used to guarantee a proper value of $\alpha$. However, these methods are computationally expensive and typically have not been used in the context of optimizing a PES. The recent method of Lavor ${ }^{9}$ uses interval arithmetic to obtain the lower bounds on the potential energy. This approach provides a mathematical and computational 
guarantee that the lower bounds are valid, but is still quite expensive computationally.

We describe here a new methodology for the deterministic global minimization of a potential energy surface. Like Lavor's method, ${ }^{9}$ it is based on interval mathematics. However, the use of an interval-Newton/generalized-bisection (IN/GB) strategy for pruning subdomains, and of Taylor models for bounding the potential energy, makes this new method orders of magnitude more efficient than the method of Lavor. ${ }^{9}$ This is demonstrated using the molecular structure problems considered by Lavor, ${ }^{9}$ as well as with an additional group of problems. The second group of problems involves a more realistic potential energy function, representative of those in current use, and demonstrates the direct applicability of the new methodology in computational chemistry.

In the next section we provide a brief background in interval analysis, and in Section 3 we give details of the new methodology including the interval-Newton technique and some recent improvements in it, and the use of Taylor models. Examples and performance results are then presented in Section 4.

\section{Interval analysis}

A real interval $X$ is defined as the set of real numbers lying between (and including) given upper and lower bounds; that is, $X=[\underline{X}, \bar{X}]=\{x \in \Re \mid \underline{X} \leq x \leq \bar{X}\}$. Here an underline is used to indicate the lower bound of an interval and an overline is used to indicate the upper bound. A real interval vector $\boldsymbol{X}=\left(X_{1}, X_{2}, \ldots, X_{n}\right)^{\mathrm{T}}$ has $n$ real interval components and can be interpreted geometrically as an $n$-dimensional rectangle or box. Note that in this context uppercase quantities are intervals, and lowercase quantities or uppercase quantities with underline or overline are real numbers.

Basic arithmetic operations with intervals are defined by $X$ op $Y=\{x$ op $y \mid x \in X, y \in Y\}$, 
where op $=\{+,-, \times, \div\}$. Interval versions of the elementary functions can be similarly defined. It should be emphasized that, when machine computations with interval arithmetic operations are done, as in the procedures outlined below, the endpoints of an interval are computed with a directed (outward) rounding. That is, the lower endpoint is rounded down to the next machinerepresentable number and the upper endpoint is rounded up to the next machine-representable number. In this way, through the use of interval arithmetic, as opposed to floating-point arithmetic, any potential rounding error problems are avoided. Several good introductions to interval analysis, as well as interval arithmetic and other aspects of computing with intervals, are available. ${ }^{17-20}$ Implementations of interval arithmetic and elementary functions are also readily available, and recent compilers from Sun Microsystems directly support interval arithmetic and an interval data type.

For an arbitrary function $f(\boldsymbol{x})$, the interval extension $F(\boldsymbol{X})$ encloses all possible values of $f(\boldsymbol{x})$ for $\boldsymbol{x} \in \boldsymbol{X}$; that is, it encloses the range of $f(\boldsymbol{x})$ over $\boldsymbol{X}$. It is often computed by substituting the given interval $\boldsymbol{X}$ into the function $f(\boldsymbol{x})$ and then evaluating the function using interval arithmetic. This so-called "natural" interval extension is often wider than the actual range of function values, though it always includes the actual range. For example, the natural interval extension of $f(x)=$ $x /(x-1)$ over the interval $X=[2,3]$ is $F([2,3])=[2,3] /([2,3]-1)=[2,3] /[1,2]=[1,3]$, while the true function range over this interval is $[1.5,2]$. This overestimation of the function range is due to the "dependency" problem, which may arise when a variable occurs more than once in a function expression. While a variable may take on any value within its interval, it must take on the same value each time it occurs in an expression. However, this type of dependency is not recognized when the natural interval extension is computed. In effect, when the natural interval extension is used, the range computed for the function is the range that would occur if 
each instance of a particular variable were allowed to take on a different value in its interval range. For the case in which $f(\boldsymbol{x})$ is a single-use expression, that is, an expression in which each variable occurs only once, natural interval arithmetic will always yield the true function range. For example, rearrangement of the function expression used above gives $f(x)=x /(x-1)=1+1 /(x-1)$, and now $F([2,3])=1+1 /([2,3]-1)=1+1 /[1,2]=1+[0.5,1]=[1.5,2]$, the true range. For cases in which such rearrangements are not possible, there are a variety of other approaches that can be used to try to tighten interval extensions, ${ }^{17-20}$ including the use of Taylor models, as described as part of the next section.

\section{Methodology}

Of particular interest here is the interval-Newton method. For the unconstrained minimization of $\phi(\boldsymbol{x})$, this technique provides the capability to find tight enclosures of all global minimizers in some specified initial interval $\boldsymbol{X}^{(0)}$, and to do so with mathematical and computational certainty. An outline of the interval-Newton methodology is given here. More details are available elsewhere. ${ }^{18,19}$ It should be emphasized that this technique is not equivalent to simply implementing the routine "point" Newton method in interval arithmetic.

The interval-Newton method is basically an equation-solving method. In the context of unconstrained minimization, it is used to seek solutions of $\boldsymbol{f}(\boldsymbol{x})=\nabla \phi(\boldsymbol{x})=\mathbf{0}$, where $\nabla \phi(\boldsymbol{x})$ indicates the gradient of the objective function $\phi(\boldsymbol{x})$. Given some initial interval, sufficiently large that the global minimum sought is in its interior, the interval-Newton algorithm is applied to a sequence of subintervals. For a subinterval $\boldsymbol{X}^{(k)}$ in the sequence, the first step is the function (gradient) range test. An interval extension $\boldsymbol{F}\left(\boldsymbol{X}^{(k)}\right)$ of the function $\boldsymbol{f}(\boldsymbol{x})=\nabla \phi(\boldsymbol{x})$ is calculated. If there is any component of the interval extension $\boldsymbol{F}\left(\boldsymbol{X}^{(k)}\right)$ that does not include zero, then no solution of 
$\boldsymbol{f}(\boldsymbol{x})=0$, that is, no stationary point of $\phi(\boldsymbol{x})$, can exist in this interval. This interval can then be discarded since the global minimum must be one of the stationary points. The next subinterval in the sequence may then be considered. Otherwise, testing of $\boldsymbol{X}^{(k)}$ continues. During this step, other interval-based techniques (e.g., constraint propagation) may also be applied to try to shrink $\boldsymbol{X}^{(k)}$ before proceeding. Note that we have assumed that the initial interval $\boldsymbol{X}^{(0)}$ is sufficiently large that the global minimum will not be on its boundary, since an extremum on the boundary is in general not a stationary point. For situations in which such an assumption cannot be made, the "peeling" process described by Kearfott, ${ }^{19}$ in which interval-Newton is applied to each of the lower dimensional subspaces that constitute the boundary of $\boldsymbol{X}^{(0)}$, can be used.

The next step is the objective range test. An interval extension $\Phi\left(\boldsymbol{X}^{(k)}\right)$, containing the range of $\phi(\boldsymbol{x})$ over $\boldsymbol{X}^{(k)}$ is computed. If the lower bound of $\Phi\left(\boldsymbol{X}^{(k)}\right)$ is greater than a known upper bound $\hat{\phi}$ on the global minimum (the initialization of this upper bound is discussed below), then $\boldsymbol{X}^{(k)}$ can be discarded since it cannot contain the global minimum and need not be further tested. To obtain $\Phi\left(\boldsymbol{X}^{(k)}\right)$, the natural interval extension can be used, as done by Lavor, ${ }^{9}$ but other techniques can also be employed. We will also compute $\Phi\left(\boldsymbol{X}^{(k)}\right)$ by the first order Taylor expansion of $\phi(\boldsymbol{x}),{ }^{18}$

$$
\Phi\left(\boldsymbol{X}^{(k)}\right)=\phi\left(\boldsymbol{x}_{0}^{(k)}\right)+\left(\boldsymbol{X}^{(k)}-\boldsymbol{x}_{0}^{(k)}\right)^{T} \boldsymbol{F}\left(\boldsymbol{X}^{(k)}\right)
$$

where $\boldsymbol{x}_{0}^{(k)}$ is a real point inside the interval $\boldsymbol{X}^{(k)}$. This often provides tighter bounds than the natural interval extension, especially when $\boldsymbol{X}^{(k)}$ is small, and can also be used in other ways to reduce or eliminate the current interval, as explained below. The final determination of $\Phi\left(\boldsymbol{X}^{(k)}\right)$ is then done by taking the intersection of the natural interval extension and the interval extension computed from the Taylor expansion.

The tightness of the bounds provided by the interval extension in Eq. (1) depends on the choice 
of the real point $\boldsymbol{x}_{0}^{(k)}$. Thus it is possible to manipulate $\boldsymbol{x}_{0}^{(k)}$ such that the lower bound of $\Phi\left(\boldsymbol{X}^{(k)}\right)$ is maximized. To determine exactly the optimal $\boldsymbol{x}_{0}^{(k)}$ point would require optimizing an expression involving the function $\phi(\boldsymbol{x})$. Since this is impractical in this context, we will neglect the variation of $\phi(\boldsymbol{x})$ in Eq. (1), and thus seek a point for $\boldsymbol{x}_{0}^{(k)}$ such that the lower bound on just the second term on the right hand side of Eq. (1) is maximized. That is, to choose $\boldsymbol{x}_{0}^{(k)}$ we will solve

$$
\max _{\boldsymbol{x}_{0}^{(k)} \in \boldsymbol{X}^{(k)}} \underline{\left(\boldsymbol{X}^{(k)}-\boldsymbol{x}_{0}^{(k)}\right)^{T} \boldsymbol{F}\left(\boldsymbol{X}^{(k)}\right)} .
$$

The objective function in Eq. (2) is a sum in which each term depends on only one component of $\boldsymbol{x}_{0}^{(k)}$. Thus, the problem in Eq. (2) is equivalent to the series of single-variable problems

$$
\max _{x_{0 i}^{(k)} \in X_{i}^{(k)}} \quad \underline{\left(X_{i}^{(k)}-x_{0 i}^{(k)}\right) F_{i}}, \quad i=1,2, \ldots, n .
$$

The point $\boldsymbol{x}_{0}^{(k)}$ can be now determined by solving the optimization problem (3), the solution to which is

$$
x_{0 i}^{(k)}=\frac{\overline{F_{i}} X_{i}^{(k)}-\underline{F_{i}} \overline{X_{i}^{(k)}}}{\overline{F_{i}}-\underline{F_{i}}} .
$$

While a point $\boldsymbol{x}_{0}^{(k)}$ chosen this way will not be optimal, we expect that in general it will be an improvement over just choosing $\boldsymbol{x}_{0}^{(k)}$ arbitrarily.

If at this point, $\underline{\Phi\left(\boldsymbol{X}^{(k)}\right)}<\hat{\phi}$, and thus it has not been possible to discard $\boldsymbol{X}^{(k)}$, there may be a point $\boldsymbol{x}$ in $\boldsymbol{X}^{(k)}$ for which $\phi(\boldsymbol{x})<\hat{\phi}$, allowing us to update (reduce) $\hat{\phi}$, the upper bound on the global minimum of $\phi$. However, this is not known for certain (since it is likely that $\Phi\left(\boldsymbol{X}^{(k)}\right.$ ) overestimates the true range of $\phi$ over $\boldsymbol{X}^{(k)}$ ), and thus, to reduce computational overhead, we only attempt an update of $\hat{\phi}$ if $\phi\left(\boldsymbol{x}_{0}^{(k)}\right)<\hat{\phi}$, which indicates with certainty that there is a point in $\boldsymbol{X}^{(k)}$ that can be used to update $\hat{\phi}$. So, if $\phi\left(\boldsymbol{x}_{0}^{(k)}\right)<\hat{\phi}$, a local optimization routine starting at $\boldsymbol{x}_{0}^{(k)}$ is used to find a local minimum, which then provides an updated (smaller) $\hat{\phi}$, i.e., a better upper bound 
on the global minimum. For this purpose, we use the bound-constrained quasi-Newton method L-BFGS-B. ${ }^{21}$

The updated value of $\hat{\phi}$ can then be used, together with the Taylor model for the interval extension of $\phi$, to delete part of the current subinterval, or even eliminate it entirely, since only the part of $\boldsymbol{X}^{(k)}$ (if any) for which the condition $\Phi\left(\boldsymbol{X}^{(k)}\right)-\hat{\phi} \leq 0$ is satisfied can contain the global minimum and thus needs to be kept.

For some $j \in\{1,2, \ldots, n\}$, let $U=[a, b]=\phi\left(\boldsymbol{x}_{0}^{(k)}\right)-\hat{\phi}+\sum_{i=1, i \neq j}^{n}\left(X_{i}^{(k)}-x_{0 i}^{(k)}\right) F_{i}\left(\boldsymbol{X}^{(k)}\right), V=$ $[c, d]=F_{j}\left(\boldsymbol{X}^{(k)}\right)$, and $T=X_{j}^{(k)}-x_{0 j}^{(k)}$. The part of $X_{j}^{(k)}$ containing points $x_{j}^{(k)}$ such that

$$
U+V T \leq 0
$$

needs to be retained. The set $T$ that satisfies Eq. (5), can be determined to be

$$
T=\left\{\begin{array}{ll}
{[-a / d, \infty]} & \text { if } a \leq 0 \text { and } d<0 \\
{[-a / c, \infty]} & \text { if } a>0, c<0, \text { and } d \leq 0 \\
{[-\infty, \infty]} & \text { if } a \leq 0 \text { and } c \leq d \leq 0 \\
{[-\infty,-a / d] \cup[-a / c, \infty]} & \text { if } a>0 \text { and } c<0<d \\
{[-\infty,-a / c]} & \text { if } a \leq 0 \text { and } c>0 \\
{[\infty,-a / d]} & \text { if } a>0, c \geq 0, \text { and } d>0 \\
\emptyset & \text { if } a>0 \text { and } c=d=0
\end{array} .\right.
$$

Thus, beginning with $j=1, T$ is computed and $X_{j}^{(k)}$ is replaced by $X_{j}^{(k)} \cap\left(T+x_{j}^{(k)}\right)$. If $X_{j}^{(k)}$ is now empty, the subinterval $\boldsymbol{X}^{(k)}$ can be discarded. Otherwise, the improved $X_{j}^{(k)}$ is used in computing $U=[a, b]$ and $V=[c, d]$ to find improvements of subsequent components of $\boldsymbol{X}^{(k)}$. This procedure proceeds component by component until all components of $\boldsymbol{X}^{(k)}$ have been updated or $\boldsymbol{X}^{(k)}$ has 
been discarded.

If $\boldsymbol{X}^{(k)}$ has not been eliminated in the objective range test, then the next step is the intervalNewton test. The linear interval equation system

$$
\boldsymbol{F}^{\prime}\left(\boldsymbol{X}^{(k)}\right)\left(\boldsymbol{N}^{(k)}-\tilde{\boldsymbol{x}}^{(k)}\right)=-\boldsymbol{f}\left(\tilde{\boldsymbol{x}}^{(k)}\right)
$$

is solved for a new interval $\boldsymbol{N}^{(k)}$, where $\boldsymbol{F}^{\prime}\left(\boldsymbol{X}^{(k)}\right)$ is an interval extension of the Jacobian of $\boldsymbol{f}(\boldsymbol{x})$ (i.e., an interval extension of the Hessian of $\phi(\boldsymbol{x})$ ), and $\tilde{\boldsymbol{x}}^{(k)}$ is an arbitrary point in $\boldsymbol{X}^{(k)}$. It has been shown ${ }^{18-20}$ that any root (stationary point of $\phi(\boldsymbol{x})$ ) contained in $\boldsymbol{X}^{(k)}$ is also contained in the image $\boldsymbol{N}^{(k)}$. This implies that if the intersection between $\boldsymbol{X}^{(k)}$ and $\boldsymbol{N}^{(k)}$ is empty, then no root exists in $\boldsymbol{X}^{(k)}$, and also suggests the iteration scheme $\boldsymbol{X}^{(k+1)}=\boldsymbol{X}^{(k)} \cap \boldsymbol{N}^{(k)}$. In addition, it has also been shown ${ }^{18-20}$ that, if $\boldsymbol{N}^{(k)} \subset \boldsymbol{X}^{(k)}$, then there is a unique root contained in $\boldsymbol{X}^{(k)}$ and thus in $\boldsymbol{N}^{(k)}$. Thus, after computation of $\boldsymbol{N}^{(k)}$ from Eq. (7), there are three possibilities: (1) $\boldsymbol{X}^{(k)} \cap \boldsymbol{N}^{(k)}=\emptyset$, meaning there is no root in the current interval $\boldsymbol{X}^{(k)}$ and it can be discarded; (2) $\boldsymbol{N}^{(k)} \subset \boldsymbol{X}^{(k)}$, meaning that there is exactly one root in the current interval $\boldsymbol{X}^{(k)} ;(3)$ neither of the above, meaning that no conclusion can be drawn. In the last case, if $\boldsymbol{X}^{(k)} \cap \boldsymbol{N}^{(k)}$ is sufficiently smaller than $\boldsymbol{X}^{(k)}$, then the interval-Newton test can be reapplied to the resulting intersection, $\boldsymbol{X}^{(k+1)}=\boldsymbol{X}^{(k)} \cap \boldsymbol{N}^{(k)}$. Otherwise, the intersection $\boldsymbol{X}^{(k)} \cap \boldsymbol{N}^{(k)}$ is bisected, and the resulting two subintervals are added to the sequence (stack) of subintervals to be tested. If an interval containing a unique root has been identified, then this root can be tightly enclosed by continuing the interval-Newton iteration, which will converge quadratically to a desired tolerance (on the enclosure diameter).

Clearly, the solution of the linear interval system given by Eq. (7) is essential to this approach. To see the issues involved in solving such a system, consider the general linear interval system $\boldsymbol{A} \boldsymbol{z}=\boldsymbol{B}$, where the matrix $\boldsymbol{A}$ and the right-hand-side vector $\boldsymbol{B}$ are interval-valued. The solution 
set $\mathcal{S}$ of this system is defined by $\mathcal{S}=\{\boldsymbol{z} \mid \tilde{A} \boldsymbol{z}=\boldsymbol{b}, \tilde{A} \in \boldsymbol{A}, \boldsymbol{b} \in \boldsymbol{B}\}$. However, in general this set is not an interval and may have a very complex, polygonal geometry. Thus to "solve" the linear interval system, one instead seeks an interval $\boldsymbol{Z}$ containing $\mathcal{S}$. Computing the interval hull (the tightest interval containing $\mathcal{S}$ ) is NP-hard, ${ }^{22}$ but there are several methods for determining an interval $\boldsymbol{Z}$ that contains but overestimates $\mathcal{S}$. Various interval-Newton methods differ in how they solve Eq. (7) for $\boldsymbol{N}^{(k)}$ and thus in the tightness with which the solution set is enclosed. By obtaining bounds that are as tight as possible, the overall performance of the interval-Newton approach can be improved, since with a smaller $\boldsymbol{N}^{(k)}$ the volume of $\boldsymbol{X}^{(k)} \cap \boldsymbol{N}^{(k)}$ is reduced, and it is also more likely that either $\boldsymbol{X}^{(k)} \cap \boldsymbol{N}^{(k)}=\emptyset$ or $\boldsymbol{N}^{(k)} \subset \boldsymbol{X}^{(k)}$ will be satisfied. Thus, intervals that may contain solutions of the nonlinear system are more quickly contracted, and intervals that contain no solution or that contain a unique solution may be more quickly identified, all of which leads to a likely reduction in the number of bisections needed.

Frequently, $\boldsymbol{N}^{(k)}$ is computed component-wise using an interval Gauss-Seidel approach, preconditioned with an inverse-midpoint matrix. Though the inverse-midpoint preconditioner is a good general-purpose preconditioner, it is not always the most effective approach. ${ }^{19}$ Recently, a hybrid preconditioning approach (HP/RP), which combines a simple pivoting preconditioner (and a method for selecting the real point $\left.\tilde{\boldsymbol{x}}^{(k)}\right)$ with the standard inverse-midpoint scheme, has been described by Gau and Stadtherr ${ }^{23}$ and shown to achieve substantially more efficient computational performance than the inverse-midpoint preconditioner alone, in some cases by multiple orders of magnitude. However, it still cannot yield the tightest enclosure of the solution set, which, as noted above, is in general an NP-hard problem. Lin and Stadtherr ${ }^{24,25}$ have recently suggested a strategy (LISS_LP) based on linear programming (LP) for solving the linear interval system, Eq. (7), arising in the context of interval-Newton methods. Using this approach, exact component-wise bounds on 
the solution set can be calculated, while avoiding exponential time complexity. In numerical experiments, ${ }^{24,25}$ LISS_LP has been shown to achieve further computational performance improvements compared with HP/RP.

This approach, as outlined above, is referred to as an interval-Newton/generalized-bisection (IN/GB) method. At termination, when the subintervals in the sequence have all been tested, all global minimizers will have been tightly enclosed. It can be regarded as a type of branchand-bound scheme on a binary tree. Although the interval-Newton test is effective in reducing and eliminating subdomains from the search space, it is also relatively expensive computationally. Thus, in considering the overall computation time, one must consider the trade-off between the expense of the interval-Newton step and the reduction of the search space that it provides. That is, in some problems, use of the interval-Newton step may drastically reduce the overall computation time, but, in other problems, even though it reduces the number of subdomains that must be considered, use of the interval-Newton test may actually increase the overall computation time. This trade-off will be studied in the examples presented below.

As with any type of procedure incorporating branch-and-bound, an important issue in implementing the methodology described above is how to initialize $\hat{\phi}$, the upper bound on the global minimum. There are many ways in which this can be done, and clearly it is desirable to find a $\hat{\phi}$ that is as small as possible (i.e., tightest possible upper bound). To initialize $\hat{\phi}$ for the type of problem studied here, in which there are an extremely large number of local minima, we run $n^{2}$ local minimizations ( $n$ is the number of variables) using a BFGS algorithm from randomly chosen starting points, and then choose the smallest value of $\phi$ found to be the initial $\hat{\phi}$. 


\section{Examples: Optimization of molecular structures}

\subsection{Example 1}

This is a simplified molecular model studied by Lavor. ${ }^{9}$ It was chosen for use as an example here so that we can make direct comparisons between the performance of the methodology presented above and the interval-based method given by Lavor. ${ }^{9}$ Following Lavor, we consider a linear chain of $N$ "beads" (united atoms) centered at $\boldsymbol{x}_{1}, \boldsymbol{x}_{2}, \ldots, \boldsymbol{x}_{N}$ in a three dimensional space. The total potential energy can be represented as

$$
\mathcal{V}=\mathcal{V}_{1}+\mathcal{V}_{2}+\mathcal{V}_{3}+\mathcal{V}_{4}
$$

where $\mathcal{V}_{1}$ is the contribution due to bond stretching for every pair of consecutive beads,

$$
\mathcal{V}_{1}=\sum_{(i, j) \in M_{1}} c_{i j}^{(1)}\left(r_{i j}-r_{i j}^{0}\right)^{2}
$$

with $c_{i j}^{(1)}$ the bond stretching force constant and $r_{i j}^{0}$ the equilibrium bond length; $\mathcal{V}_{2}$ is the contribution due to bond angle bending over every three consecutive beads,

$$
\mathcal{V}_{2}=\sum_{(i, j) \in M_{2}} c_{i j}^{(2)}\left(\theta_{i j}-\theta_{i j}^{0}\right)^{2}
$$

with $c_{i j}^{(2)}$ the angle bending force constant and $\theta_{i j}^{0}$ the equilibrium bond angle; $\mathcal{V}_{3}$ is the contribution due to the dihedral angle over every four consecutive beads,

$$
\mathcal{V}_{3}=\sum_{(i, j) \in M_{3}} c_{i j}^{(3)}\left(1+\cos \left(3 \omega_{i j}-\omega_{i j}^{0}\right)\right)
$$

with $c_{i j}^{(3)}$ the torsion force constant and $\omega_{i j}^{0}$ the equilibrium angle; and $\mathcal{V}_{4}$ is the potential that characterizes the two-body interactions between every pair of beads separated by more than three covalent bonds along the chain. Here only interactions between pairs separated by exactly three 
covalent bonds are considered, and the model used is

$$
\mathcal{V}_{4}=\sum_{(i, j) \in M_{3}} \frac{(-1)^{i}}{r_{i j}}
$$

with $r_{i j}$ the distance separating beads $i$ and $j$. In the expressions above, the notation $M_{i}, i=1,2,3$, indicates the sets of pairs of beads separated by $i$ covalent bonds.

In this problem, all covalent bond lengths and bond angles are fixed at the equilibrium values $r_{i j}^{0}=1.526 \AA$ and $\theta_{i j}^{0}=1.91$ (characteristic of a hydrocarbon chain). Also the values $c_{i j}^{(3)}=1$ and $\omega_{i j}^{0}=0$ are set. The potential energy function then becomes

$$
\mathcal{V}=\mathcal{V}_{3}+\mathcal{V}_{4}=\sum_{(i, j) \in M_{3}}\left[1+\cos \left(3 \omega_{i j}\right)\right]+\sum_{(i, j) \in M_{3}} \frac{(-1)^{i}}{r_{i j}}
$$

where $r_{i j}$ can be expressed as a function of dihedral angle using 9

$$
r_{i j}=\sqrt{10.60099896-4.14720682 \cos \left(\omega_{i j}\right)} \quad(i, j) \in M_{3} .
$$

The problem is then to find the global minimum of this potential function, which is now a function only of the dihedral angles $\omega_{i j},(i, j) \in M_{3}$. The initial intervals for the search are set as $\omega_{i j} \in[0,5]$ for all $(i, j) \in M_{3}$, as Lavor $^{9}$ also did. The global minimizer of Eq. (13) can be determined analytically $^{26}$ for the $N$-atom case, and is the alternating sequence of dihedral angles $\omega_{i, i+3}=$ 1.039195303 and $\omega_{i+1, i+4}=\pi, i=1,3,5, \ldots, N$. This simplified molecular model is a very good test problem thanks to the known global minimum and the huge number $\left(2^{N-3}\right)$ of local minima that are present.

The interval-Newton methodology described above was successfully applied to solve a set of problems with different chain length $N$, with the correct global minimizer found in each case. Table 1 shows the global minimum energy value found, along with computational performance results on an Intel Pentium 4 3.2Ghz machine. In order to study the trade-offs involved in using 
the interval-Newton (I-N) test, results are shown both with the I-N test and with the I-N test turned off. Clearly, use of the interval-Newton test results in a dramatic improvement in overall computation time for this problem. The largest problem tested was $N=40$, for which it took about 3499 seconds to find the global minimum. In Lavor's study, ${ }^{9}$ in which the methodology used incorporated only the function (gradient) range test and the objective range test with natural interval extension, the largest problem solved was $N=25$, which required 34657 seconds CPU time on a Pentium III 700MHz machine. Using our methodology, with the interval-Newton test, 8.3 seconds was required to solve this problem on a Intel Pentium $43.2 \mathrm{GHz}$ machine. Based on SPEC benchmark results, ${ }^{27}$ a Pentium $43.2 \mathrm{GHz}$ machine is about 6 times faster than a Pentium III 700Mhz machine. Thus, the new methodology described here appears to be multiple orders of magnitude more efficient than the method of Lavor. ${ }^{9}$ The greatly improved performance can be attributed to the use of the interval-Newton procedure, together with the use of a Taylor model for bounding the objective function.

\subsection{Example 2}

This second set of example problems involves a much more complex and realistic model ${ }^{28}$ of $n$-alkanes than considered by Lavor. ${ }^{9}$ The model employs potential functions commonly used in computational chemistry today, and thus allows us to explore the applicability of the interval methodology in this context. The $n$-alkane molecule is assumed to consist of the appropriate number of united atoms, representing both the methyl and methylene groups along the backbone of the alkane molecule. Following Maginn et al. ${ }^{28}$ bond lengths are assumed rigid and fixed at the equilibrium value $r_{i j}^{0}=1.53 \AA$, and bond angles between three adjacent united atoms are fixed to the equilibrium value, $\theta^{0}=112^{\circ}$. 
For alkanes, the torsion (dihedral) potential, which governs rotation around nonterminal carboncarbon bonds, is often described ${ }^{29}$ using the Ryckaert-Bellemans (RB) potential, ${ }^{30}$ a six-term expansion $v_{t}\left(\omega_{i}\right)$ in the cosine of each dihedral angle,

$$
\mathcal{V}_{3}=\sum_{i=4}^{N} v_{t}\left(\omega_{i}\right)=\sum_{i=4}^{N} \sum_{j=0}^{5} C_{j}\left(\cos \omega_{i}\right)^{j}
$$

where $N$ is the number of atoms. Here, the notation used is $\omega_{i}=\omega_{i-3, i-2, i-1, i}$, the dihedral angle involving atoms $i-3, i-2, i-1$, and $i$. The RB potential includes, as a special case $\left(C_{4}=C_{5}=0\right)$, the popular OPLS dihedral potential, ${ }^{29,31}$ which is often expressed in terms of a Fourier series equivalent to Eq. (15). In other popular force fields, ${ }^{32}$ a less complex expression for the torsion potential, involving perhaps only a single cosine term, such as Eq. (11), may be used. For this example, we will use the full RB potential as given in Eq. (15).

For $n$-alkane molecules longer than butane, additional intramolecular potential terms are necessary to represent the van der Waals interactions of atoms separated by more than three carboncarbon bonds. For these nonpolar molecules, Coulombic interactions are treated as negligible. For the van der Waals interactions, a standard Lennard-Jones 6-12 potential is used. This is given by

$$
\nu_{4}=\sum_{i=1}^{N-4} \sum_{j=i+4}^{N} v_{L J}\left(r_{i j}\right)=\sum_{i=1}^{N-4} \sum_{j=i+4}^{N} 4 \epsilon_{i j}\left[\left(\frac{\sigma_{i j}}{r_{i j}}\right)^{12}-\left(\frac{\sigma_{i j}}{r_{i j}}\right)^{6}\right]
$$

where $r_{i j}$ is the distance between two interacting atoms $i$ and $j$, and is a function of the Cartesian coordinates of the atoms in the molecule,

$$
r_{i j}^{2}=\left(x_{j}-x_{i}\right)^{2}+\left(y_{j}-y_{i}\right)^{2}+\left(z_{j}-z_{i}\right)^{2}
$$

Thompson ${ }^{33}$ has provided an expression for the Cartesian coordinates of an atom as functions of bond lengths, covalent bond angles and dihedral angles. If the first three atoms in the chain are represented by the coordinates, $(0,0,0)^{T},\left(-r_{2}, 0,0\right)^{T}$, and $\left(r_{3} \cos \left(\theta_{3}\right)-r_{2}, r_{3} \sin \left(\theta_{3}\right), 0\right)^{T}$, respectively, 
then, for $4 \leq k \leq N$, the coordinates of atom $k$ in an $N$-atom acyclic chain are

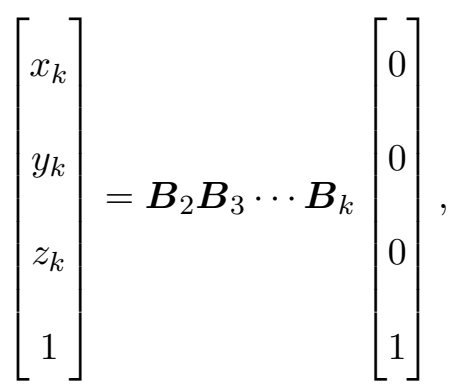

where $\boldsymbol{B}_{2}, \boldsymbol{B}_{3}$, and $\boldsymbol{B}_{k}$ are the $4 \times 4$ transformation matrices

$$
\begin{aligned}
& \boldsymbol{B}_{2}=\left[\begin{array}{cccc}
-1 & 0 & 0 & -r_{2} \\
0 & 1 & 0 & 0 \\
0 & 0 & -1 & 0 \\
0 & 0 & 0 & 1
\end{array}\right] \\
& \boldsymbol{B}_{3}=\left[\begin{array}{cccc}
-\cos \left(\theta_{3}\right) & -\sin \left(\theta_{3}\right) & 0 & -r_{3} \cos \left(\theta_{3}\right) \\
\sin \left(\theta_{3}\right) & -\cos \left(\theta_{3}\right) & 0 & -r_{3} \sin \left(\theta_{3}\right) \\
0 & 0 & 1 & 0 \\
0 & 0 & 0 & 1
\end{array}\right] \\
& \boldsymbol{B}_{k}=\left[\begin{array}{cccc}
-\cos \left(\theta_{k}\right) & -\sin \left(\theta_{k}\right) & 0 & -r_{k} \cos \left(\theta_{k}\right) \\
\sin \left(\theta_{k}\right) \cos \left(\omega_{k}\right) & -\cos \left(\theta_{k}\right) \cos \left(\omega_{k}\right) & -\sin \left(\omega_{k}\right) & -r_{k} \sin \left(\theta_{k}\right) \cos \left(\omega_{k}\right) \\
\sin \left(\theta_{k}\right) \sin \left(\omega_{k}\right) & -\cos \left(\theta_{k}\right) \sin \left(\omega_{k}\right) & \cos \left(\omega_{k}\right) & -r_{k} \sin \left(\theta_{k}\right) \sin \left(\omega_{k}\right) \\
0 & 0 & 0 & 1
\end{array}\right] .
\end{aligned}
$$

Here the notation used for the bond lengths is $r_{k}=r_{k-1, k}$, the bond length involving atoms $k-1$ and $k$, and for the bond angles, $\theta_{k}=\theta_{k-2, k-1, k}$, the bond angle involving atoms $k-2, k-1$, and $k$. As noted above, these bond lengths and angles have been fixed to equilibrium values. Thus, the total potential

$$
v=V_{3}+V_{4}
$$


is a function only of the dihedral angles, $\omega_{i}, i=4, \ldots, N$. The problem is then to find the global minimum of Eq. (22) with respect to these dihedral angles. The potential energy function used in this problem is a Class I additive function, in a form that is commonly in use today. ${ }^{32}$

All parameters in the potential function are taken from Maginn et al. ${ }^{28}$ and are summarized in Table 2. Note that, in order to conform to the common convention that the dihedral angle is $\pi$ in the trans configuration, the signs of some parameters in the torsion energy function, namely $C_{1}$, $C_{3}$, and $C_{5}$, have been changed (Maginn et al. ${ }^{28}$ took the dihedral angle to be zero in the trans configuration). Methyl and methylene groups are modeled with identical parameters.

The interval methodology will be applied to determine the global minimum in the potential energy function described above. To achieve tighter interval extensions of the potential function and its derivatives, and thus improve the performance of the methodology, the mathematical properties of the potential function are exploited. Consider first the torsion potential $\mathcal{V}_{3}$, as given by Eq. (15). Each term is given by an expression of the form

$$
v_{t}(\omega)=\sum_{j=0}^{5} C_{j}(\cos \omega)^{j}
$$

in which the dihedral angle $\omega$ appears five times. In this case, when interval arithmetic is applied to compute the interval extension, overestimation of the range will occur due to the multiple occurrence of $\omega$. However, the properties of the torsion potential can be exploited to avoid this problem, and to compute exact (within roundout) bounds on the range of the torsion potential. Figure 1 shows the torsion potential $v_{t}(\omega)$ between $\omega=0$ and $\omega=2 \pi$. There exist four maxima, at $0, \max _{1} \approx 2 \pi / 3, \max _{2} \approx 4 \pi / 3$ and $2 \pi$, and three minima at $\min _{1} \approx \pi / 3, \pi$ and $\min _{2} \approx 5 \pi / 3$. Note that these local minima and maxima are intended ${ }^{30}$ to be exactly $\pi / 3,2 \pi / 3$, etc. However, the actual locations of these stationary points are slightly different since the model parameters 
represent roundings; for example, $\min _{1} \approx 1.04704374$ but $\pi / 3 \approx 1.04719755$. In this work, the exact values (very narrow interval enclosures) of the extrema can be easily obtained by the IN/GB method to locate all stationary points of the torsion potential.

For a given dihedral angle interval $\Omega=[\underline{\Omega}, \bar{\Omega}]$, the potentials at the end points, $v_{t}(\bar{\Omega})$ and $v_{t}(\underline{\Omega})$ are first computed. Then the interval extension of the potential $v_{t}(\omega)$, namely $V_{t}(\Omega)=\left[\underline{V_{t}}, \overline{V_{t}}\right]$, can be obtained from

$$
\underline{V_{t}}= \begin{cases}v_{t}(\pi) & \underline{\Omega} \leq \pi \leq \bar{\Omega} \\ \min \left(v_{t}\left(\min _{1}\right), v_{t}(\bar{\Omega}), v_{t}(\underline{\Omega})\right) & \underline{\Omega} \leq \min _{1} \leq \bar{\Omega}<\pi \\ \min \left(v_{t}\left(\min _{2}\right), v_{t}(\bar{\Omega}), v_{t}(\underline{\Omega})\right) & \pi<\underline{\Omega} \leq \min _{2} \leq \bar{\Omega} \\ \min \left(v_{t}(\bar{\Omega}), v_{t}(\underline{\Omega})\right) & \text { otherwise }\end{cases}
$$

and

$$
\overline{V_{t}}= \begin{cases}\max \left(v_{t}\left(\max _{1}\right), v_{t}(\bar{\Omega}), v_{t}(\underline{\Omega})\right) & \underline{\Omega} \leq \max _{1} \leq \bar{\Omega} \\ \max \left(v_{t}\left(\max _{2}\right), v_{t}(\bar{\Omega}), v_{t}(\underline{\Omega})\right) & \underline{\Omega} \leq \max _{2} \leq \bar{\Omega} \\ \max \left(v_{t}(\bar{\Omega}), v_{t}(\underline{\Omega})\right) & \text { otherwise }\end{cases}
$$

Note that in implementing Eqs. (24) and (25) interval arithmetic is used to guarantee computationally rigorous bounds. For example, $v_{t}(\underline{\Omega})$ and $v_{t}(\bar{\Omega})$ are computed using interval arithmetic. Each term $v_{t}\left(\omega_{i}\right)$ in the total torsion potential $\mathcal{V}_{3}$ can be bounded in this way, resulting in the exact (within the roundout of interval arithmetic) interval extension of the torsion potential. A similar procedure has also been used to calculate the exact interval extensions of the first and second order derivatives of $v_{t}(\omega)$.

The dependency problem of interval arithmetic can also be avoided in computing the interval extension of the Lennard-Jones terms in the expression for $\mathcal{V}_{4}$, as given by Eq. (16). This is done ${ }^{34}$ 
by rearranging $v_{L J}\left(r_{i j}\right)$ into a single-use expression:

$$
v_{L J}\left(r_{i j}\right)=4 \epsilon_{i j}\left[\left(\frac{\sigma_{i j}}{r_{i j}}\right)^{12}-\left(\frac{\sigma_{i j}}{r_{i j}}\right)^{6}\right]=4 \epsilon_{i j}\left[\left(\frac{\sigma_{i j}}{r_{i j}}\right)^{6}-\frac{1}{2}\right]^{2}-\epsilon_{i j} .
$$

For a single-use expression, the natural interval extension computed using interval arithmetic will provide exact bounds. Exact interval extensions of the first and second order derivatives of the Lennard-Jones potential $v_{L J}\left(r_{i j}\right)$ can also be determined, as explained in detail by Lin and Stadtherr. ${ }^{34}$

Two versions of the interval methodology were applied to determine the global minimum in the potential energy function described above, using initial intervals of $[0,2 \pi]$ for each dihedral angle. In the first version, all test steps, including the function (gradient) range test, the objective range test with Taylor model, and the interval-Newton test, were used. In the second version, the interval-Newton test is turned off. Using these two different versions allows us to study the trade-offs involved in the use of the interval-Newton step, as described above.

A set of global optimization problems with different numbers of carbon atoms, from $N=4$ (butane) to $N=11$ (undecane), was used. In each case, and with both versions of the methodology, the global optimally dihedral angles were found to be $\omega_{i}=\pi, i=4, \ldots, N$. This is consistent with known results for $n$-alkanes, obtained using various other potential models and nondeterministic optimization methods (only for longer chains will the molecule fold back).

Computational performance results on an Intel Pentium $43.2 \mathrm{GHz}$ machine are summarized in Table 3, which gives both the CPU time and the number of subintervals that needed to be tested. As can be seen, the number of subintervals is reduced when the interval-Newton test is turned on, but only by a relatively small amount, and with the percent reduction becoming smaller with increasing number of atoms. Thus, on these problems, unlike the case of Example 1, the interval- 
Newton test provides little benefit, and the overhead in implementing it drags down the overall performance (CPU time) for $N \geq 7$.

The increase in CPU time with problem size seen in Table 3, as well as in Table 1, clearly reflects the NP-hard character of deterministic global optimization. Nevertheless, even for the realistically complex potential function considered here, there are a large number of interesting molecular structure problems that fall within the size range of the problems solved deterministically. It should also be noted that the point at which the methodology will become ineffective due to excessive computation time requirements will vary from problem to problem. In other types of applications, this methodology has been used to solve problems with over 200 degrees of freedom. ${ }^{35}$

\section{Concluding Remarks}

We have demonstrated here a new methodology for reliably predicting molecular structures from a potential energy model. The technique is based on interval analysis, in particular an intervalNewton/generalized-bisection algorithm. The approach provides a mathematical and computational guarantee that the molecular conformation with the global minimum potential energy will be found. Using the relatively simple potential model employed by Lavor, ${ }^{9}$ problems with up to 40 atoms were efficiently solved. This represents an improvement of multiple orders of magnitude over the interval-based method of Lavor. ${ }^{9}$ Using a more complex model of $n$-alkanes, representative of potential functions commonly used in computational chemistry today, problems with up to 11 atoms were solved deterministically, demonstrating the applicability of the interval methodology in this context. 


\section{Acknowledgments}

This work has been supported in part by the donors of The Petroleum Research Fund, ad-

ministered by the ACS, under Grant 35979-AC9, and by the Indiana 21st Century Research \& Technology Fund. 


\section{References}

1. T. Brodmeier and E. Pretsch, J. Comput. Chem., 15, 588-595 (1994).

2. M. A. Moret, P. G. Pascutti, P. M. Bisch, and K. C. Mundim, J. Comput. Chem., 19, 647-657 (1998).

3. Z. Li and J. A. Scheraga, J. Mol. Struct., 179, 333-352 (1988).

4. L. Piela, J. Kostrowicki, and J. A. Scheraga, J. Phys. Chem., 93, 3339-3346 (1989).

5. J. Pillardy and L. Piela, J. Phys. Chem., 99, 11805-11812 (1995).

6. C. A. Floudas, Deterministic Global Optimization: Theory, Methods and Applications, Kluwer Academic Publishers, Dordrecht, The Netherlands, 1999.

7. C. D. Maranas and C. A. Floudas, J. Global Optim., 4, 135-170 (1994).

8. J. L. Klepeis, I. P. Androulakis, M. G. Ierapetritou, and C. A. Floudas, Comput. Chem. Eng., 22, 765-788 (1998).

9. C. Lavor, Int. J. Quantum Chem., 95, 336-343 (2003).

10. A. R. Leach In Reviews in Computational Chemistry, Vol. II, pages 1-55. VCH Publishers, 1991.

11. H. A. Scheraga In Reviews in Computational Chemistry, Vol. III, pages 73-142. VCH Publishers, 1992.

12. A. Neumaier, SIAM Rev., 39, 407-460 (1997). 
13. C. A. Floudas, J. L. Klepeis, and P. M. Pardalos In F. Roberts, Ed., DIMACS Series in Discrete Mathematics and Theoretical Computer Science, Vol. 47, pages 141-171. Amer. Math. Soc., 1999.

14. D. J. Wales and H. A. Scheraga, Science, 285, 1368-1372 (1999).

15. C. S. Adjiman, S. Dallwig, C. A. Floudas, and A. Neumaier, Comput. Chem. Eng., 22, 1137 (1998).

16. C. S. Adjiman, I. P. Androulakis, and C. A. Floudas, Comput. Chem. Eng., 22, 1159 (1998).

17. L. Jaulin, M. Kieffer, O. Didrit, and É. Walter, Applied Interval Analysis, Springer-Verlag, London, 2001.

18. E. Hansen and G. W. Walster, Global Optimization Using Interval Analysis, Marcel Dekker, New York, 2004.

19. R. B. Kearfott, Rigorous Global Search Continuous Problems, Kluwer Academic Publishers, Dordrecht, The Netherlands, 1996.

20. A. Neumaier, Interval Methods for Systems of Equations, Cambridge University Press, Cambridge, England, 1990.

21. R. H. Byrd, P. Lu, J. Nocedal, and C. Zhu, SIAM J. Sci. Comput., 16, 1190-1208 (1995).

22. J. Rohn and V. Kreinovich, SIAM J. Matrix. Anal., 16, 415-420 (1995).

23. C. Y. Gau and M. A. Stadtherr, Comput. Chem. Eng., 26, 827-840 (2002).

24. Y. Lin and M. A. Stadtherr, J. Global Optim., 29, 281-296 (2004). 
25. Y. Lin and M. A. Stadtherr, Ind. Eng. Chem. Res., 43, 3741-3749 (2004).

26. C. Lavor A Deterministic Approach for Global Minimization of Potential Energy of Molecules PhD thesis, Federal University of Rio de Janeiro, (2001).

27. Standard Performance Evaluation Corporation. (http://www.specbench.org).

28. E. J. Maginn, A. T. Bell, and D. N. Theodorou, J. Phys. Chem., 99, 2057-2079 (1995).

29. D. van der Spoel, E. Lindahl, B. Hess, A. R. van Buuren, E. Apol, P. J. Meulenhoff, D. P. Tieleman, A. L. T. M. Sijbers, K. A. Feenstra, R. van Drunen, and H. J. C. Berendsen, Gromacs User Manual version 3.2, www.gromacs.org, 2004.

30. J. P. Ryckaert and A. Bellemans, Faraday Discuss. Chem. Soc., 66, 95-106 (1978).

31. W. L. Jorgensen, J. D. Madura, and C. J. Swenson, J. Am. Chem. Soc., 106, 6638-6646 (1984).

32. A. D. MacKerell, J. Comput. Chem., 25, 1584-1604 (2004).

33. H. B. Thompson, J. Chem. Phys., 47, 3407 (1969).

34. Y. Lin and M. A. Stadtherr, J. Chem. Phys., 121, 10159-10166 (2004).

35. C. Y. Gau and M. A. Stadtherr, AIChE J., 48, 1191-1197 (2002). 
Table 1: Computational results for Example 1

\begin{tabular}{lccc}
\hline \hline & & \multicolumn{2}{c}{ CPU time (s) } \\
\cline { 3 - 4 }$N$ & Global Minimum & with I-N test & without I-N test \\
\hline 5 & -0.08224 & 0.0009 & 0.003 \\
10 & -0.58939 & 0.02 & 0.05 \\
15 & -0.49342 & 0.16 & 0.55 \\
20 & -1.00057 & 1.53 & 9.89 \\
25 & -0.90460 & 8.31 & 168.5 \\
30 & -1.41175 & 76.02 & $>3600$ \\
35 & -1.31579 & 396.2 & - \\
40 & -1.82294 & 3499.5 & - \\
\hline
\end{tabular}


Table 2: Potential energy model parameters for Example 2

\begin{tabular}{cc}
\hline \hline Parameter & Value \\
\hline$\epsilon_{i j}$ & $72.0 \mathrm{~K}$ \\
$\sigma_{i j}$ & $3.923 \AA$ \\
$C_{0}$ & $1116 \mathrm{~K}$ \\
$C_{1}$ & $-1462 \mathrm{~K}$ \\
$C_{2}$ & $-1578 \mathrm{~K}$ \\
$C_{3}$ & $368 \mathrm{~K}$ \\
$C_{4}$ & $3156 \mathrm{~K}$ \\
$C_{5}$ & $3788 \mathrm{~K}$ \\
\hline
\end{tabular}


Table 3: Computational results for Example 2

\begin{tabular}{|c|c|c|c|c|c|}
\hline \multirow[b]{2}{*}{$N$} & \multirow[b]{2}{*}{ Global Minimum (K) } & \multicolumn{2}{|c|}{ CPU time (s) } & \multicolumn{2}{|c|}{ Subintervals tested } \\
\hline & & with I-N test & without I-N test & with I-N test & without I-N test \\
\hline 4 & 0.000000 & 0.0004 & 0.0004 & 4 & 10 \\
\hline 5 & -48.38994 & 0.004 & 0.004 & 19 & 29 \\
\hline 6 & -111.25148 & 0.02 & 0.02 & 58 & 80 \\
\hline 7 & -179.41429 & 0.13 & 0.08 & 164 & 204 \\
\hline 8 & -249.64596 & 1.82 & 0.79 & 1426 & 1508 \\
\hline 9 & -320.83597 & 20.7 & 8.11 & 10502 & 10626 \\
\hline 10 & -392.49163 & 517.0 & 182.6 & 173287 & 173492 \\
\hline 11 & -464.39913 & - & 6104.3 & - & 4285802 \\
\hline
\end{tabular}




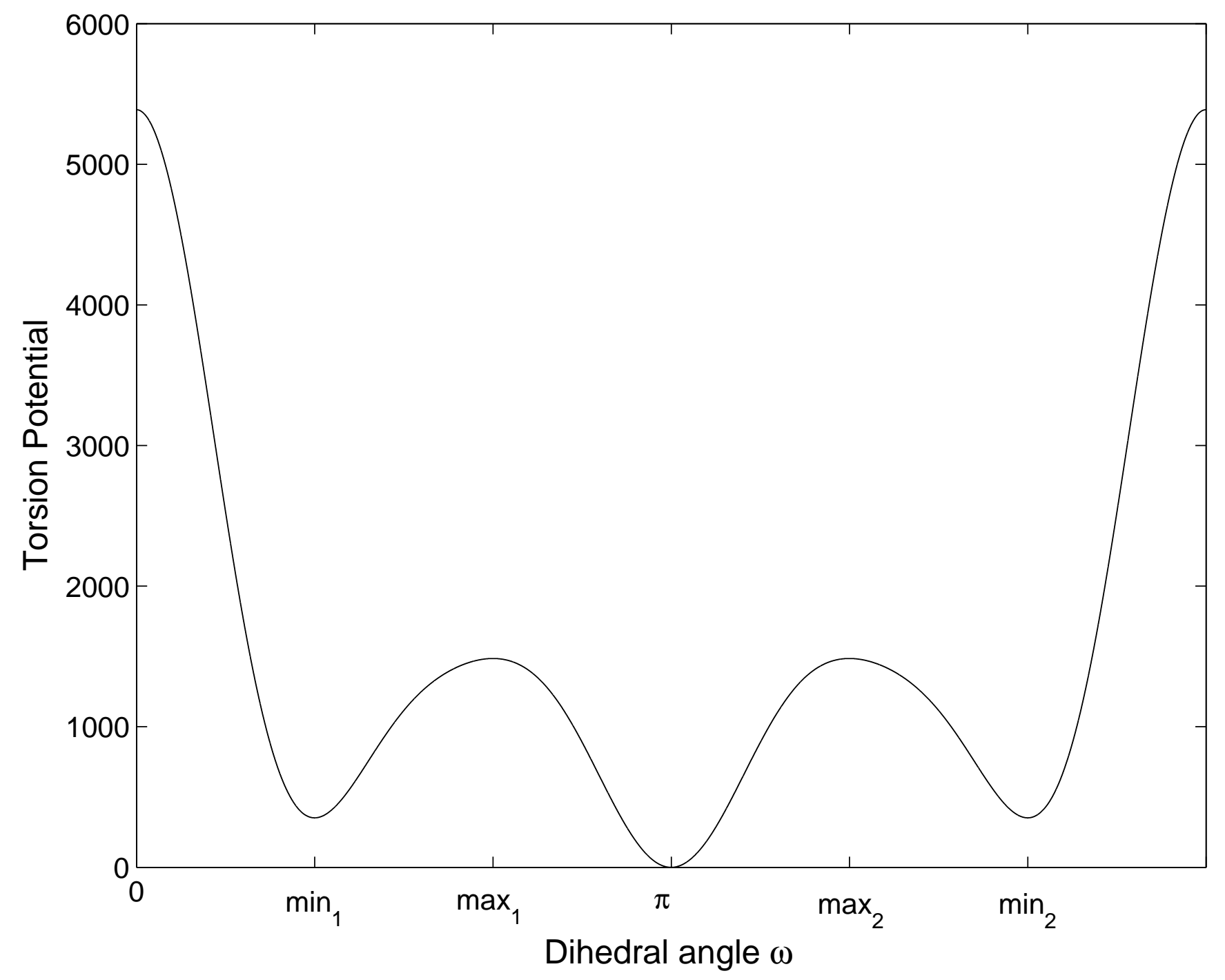

Figure 1: The torsion potential function $v_{t}(\omega)$ used in Example 2 (six-term expansion in the cosine of the dihedral angle $\omega$ ) 